\title{
Perioperative assessment of changes in tissue factor and its inhibitor in patients with abdominal aortic aneurysm treated using classical and intravascular methods
}

Objectives: We examined plasma concentrations of essential regulators of coagulation cascade, tissue factor (TF) and tissue factor inhibitor pathway (TFPI) in patients with abdominal aortic aneurysm (AAA) during two types of surgical repair, open (OAR) and endovascular repair (EVAR).

Background: The interplay between TF and TFPI is important for the proper functioning of coagulation cascade. Although abnormal coagulation is known to contribute to the formation of AAA, little is known about the prognostic value of TF and TFPI levels in the perioperative stage of AAA repair.

Methods: We have selected the reference group of 53 healthy, first-time blood donors and 66 patients scheduled for AAA repair using EVAR or OAR and measured TF and TFPI in their blood plasma using ELISA (enzyme-linked immunosorbent assay) before surgery, immediately after and 24 hours after surgery.

Results: Patients with AAA had elevated levels of TF $(219.242 \mathrm{pg} / \mathrm{ml})$ compared to the reference group (149.170 pg/ml). TF levels were higher in AAA patients with limb ischemia $(p=0.0026)$ and mural thrombi $>30 \mathrm{~mm}(p=0.0015)$ but decreased in patient with $B M I>25(p=0.027)$. No differences were observed in TF and TFPI levels during EVAR and OAR or de-pending on the stent graft type. However, there was a correlation between the TF level and occurrence of post-surgical complications in OAR type of surgery

Conclusions: Our results suggest that monitoring levels of TF and TFPI in perioperative period would be useful in risk assessment of surgery complications, especially in AAA patients with ischemic disorders undergoing OAR surgery.

Keywords: Tissue factor • tissue factor pathway inhibitor • abdominal aortic aneurysm • surgery complications

\section{Introduction}

Abdominal aortic aneurysm (AAA) is the most common type of aneurysm in aging populations across the world, predominant in males over 65 years of age. Since the etiology of AAA is still unknown and the disease is mostly asymptomatic, the risk of perforation is difficult to predict. At present, surgery remains the only treatment for AAA and the list of perioperative complications associated with the operation, such as bleeding, cardiac, renal, cerebrovascular and intestinal ischaemia, makes it a high risk procedure particularly in elderly patients. The incidence
Norbert Zapotoczny ${ }^{1,2 *}$, Maria Kotschy², Ryszard Grendziak ${ }^{1,2}$, Natalia Zuk², Joanna Dubis ${ }^{2}$ and Wojciech Witkiewicz ${ }^{1,2}$

'Department of Vascular Surgery, General Hospital in Wroclaw, Wroclaw, Poland

${ }^{2}$ Research and Development Center, General Hospital in Wroclaw, Wroclaw, Poland

*Author for correspondence: Tel: +713270331

E-mail:nzapotoczny@gmail.com Submitted: November 02, 2017 Accepted: December 08, 2017 Published online: December 13, 2017 
of perioperative complication is more frequent in case of classical open repair compared to less invasive endovascular aneurysm repair (EVAR) with local anesthesia.

The histopathological assessment of AAA samples revealed chronic inflammatory features, including decrease in smooth muscle cells, destruction of elastin fibers, remodeling of extra-cellular matrix and degradation of the connective tissue of the aortic wall due to proteolysis [1-3]. Inflammatory factors and progressive degradation of the extracellular matrix damage the endothelium, triggering the coagulation cascade. Such hemostatic disturbances are one of the main characteristics of AAA frequently leading to perioperative complications [4]. The coagulation cascade is activated by TF, a $47 \mathrm{kDa}$ trans membrane protein receptor for Factor VII/VIIa, constitutively expressed in cells surrounding blood vessels and certain tissues, such as heart, brain and lungs. TF is involved in proliferation and migration of endothelial cells as well as inflammation; it is crucial for the proper functioning of the mechanisms responsible for blood hemostasis and is actively involved in the coagulation system and fibrinolysis [5].

At present, there are no recommendations for routine monitoring of blood hemostasis of patients undergoing surgery. However, clinical observations of perioperative complications during elective AAA repair suggest the need for a more in-depth assessment of blood hemostasis. Since, the imbalance between inflammatory processes, coagulation and blood fibrinolysis is a wellknown feature of the abdominal aortic aneurysm, highlighting the role of the TF-mediated hemostasis and inflammation in the development of AAA is of great clinical importance. However, the behavior of coagulation cascade modulators in perioperative stage of AAA repair has not been studied previously. Therefore, our aim was to investigate the correlation between the level of TF and its inhibitor, TFPI during all stages of surgical intervention involving two kinds of surgical repair, classical open repair OAR and less invasive endovascular repair, EVAR.

\section{Materials and Methods}

\section{Selection of patients and control group}

The AAA patients scheduled for surgery in the Vascular Surgery Department in Regional Hospital in Wroclaw were recruited for our study between 2008 and 2011 . We have selected 66 patients, 4 patients refused their consent and were excluded from our project. Patients were aged 50-86 years, majority of subjects were men
$59(89.4 \%)$ with average age 70.6 years, there were 7 women (10.6\%, average age 70.6 years). Detailed characteristics of patients including accompanying diseases and risk factors are listed in Supplementary materials (Table 1). There are no universally accepted standards of physiological levels of TF and TFPI in healthy human populations; therefore we generated our reference group by measuring TF and TFPI levels in a group 53 healthy volunteers between the ages of 20 and 54. The reference group included 44 men and 9 women who reported for first blood donation at the Regional Blood Center and Blood Donation (RCKiK). For the analysis of TF and TFPI blood samples were drawn from the cephalic or basilic vein to the sodium citrate tubes (S-Monovette $2.9 \mathrm{ml}$, Sarstedt) in three time points: before surgery, immediately after surgery and 24 hours after surgery, centrifuged for $15 \mathrm{~min}$ at $2500 \mathrm{rpm}$. Plasma was aliquoted and stored at $-80^{\circ} \mathrm{C}$ until further analysis. All studies were conducted with the approval of the local Bioethics Commission (KB/ No.15/2014)

\begin{tabular}{|c|c|c|}
\hline & $\begin{array}{l}\text { n-number } \\
\text { (range) }\end{array}$ & $\%$ \\
\hline All patients & 66 & 100 \\
\hline Women/Men & $7 / 59$ & $10,6 / 89,4$ \\
\hline Age & $70,6(50-86)$ & \\
\hline Age women & 71,9 & \\
\hline Age men & 70,5 & \\
\hline Hypertension & 55 & 83,3 \\
\hline Ischaemic heart disease & 29 & 43,9 \\
\hline Heart attack & 19 & 28,8 \\
\hline Heart surgery & 16 & 24,2 \\
\hline Stroke & 8 & 12,1 \\
\hline TIA & 1 & 1,5 \\
\hline Carotid artery thrombosis & 6 & 9,1 \\
\hline Diabetes & 14 & 21,2 \\
\hline Dyslipidemia & 6 & 9,1 \\
\hline Urine track diseases & 31 & 47,0 \\
\hline Gastrointestinal disease & 27 & 40,9 \\
\hline Lung diseases & 14 & 21,2 \\
\hline Niedokrwienie kończyn & 13 & 19,7 \\
\hline BMI $>25$ & 48 & 72,7 \\
\hline EVAR & 46 & 69,7 \\
\hline Stent graft Gore Excluder & 29 & 43,9 \\
\hline Stent graft Cook Zenith & 17 & 25,8 \\
\hline Open Surgery & 19 & 28,8 \\
\hline Prosthesis straight & 14 & 21,2 \\
\hline Prosthesis forked & 5 & 7,6 \\
\hline Post-surgical complications & 14 & 21,2 \\
\hline
\end{tabular}




\section{Measurement of TF concentration in plasma}

The measurement of TF abundance in plasma of AAA patients and the reference group was carried out using with a commercially available IMUBIND Tissue Factor ELISA Kit (American Diagnostics, Stanford, USA) according to the protocol supplied by the manufacturer. Briefly, plasma samples were incubated in microwells coated with anti-human TF murine antibody captured with biotinylated secondary antibody labelled with horseradish peroxidase. Thus formed enzyme-antibody complex is detected using a TMB substrate with addition of 0.5 sulfuric acid for increased sensitivity. The TF concentration was measured using the BMG SPECTRO star Nano plate reader at the absorbance at $\lambda=450 \mathrm{~nm}$ were compared to those obtained on the standard curve.

\section{Measurement of plasma TFPI concentration}

TFPI plasma concentrations were measured using the commercially available IMUBIND TFPI ELISA Kit (American Diagnostics, Stanford, USA) with rabbit anti-human TFPI polyclonal anti-bodies according to the protocol supplied by the manufacturer. The affinity of antibody to native, complex and short form of TFPI was confirmed by Western Blot analysis (data not shown). Similarly to TF analysis, The TFPI concentration was measured using the BMG SPECTROstar Nano plate reader

\section{Statistical analysis}

Data normality was assessed using the D'agostinoPearson test. The analysis of TF and TFPI over time was performed using a Friedman multiple-repetition test with the Conover method. We checked the association between levels of TF and TFPI and viariables, such as age, aneurysm diameter, and thrombus using a Spearman correlation coefficient. The Mann-Whitney test was used to determine the association between plasma levels of TF and TFPI and accompanying diseases. TF and TFPI data are expressed in median and interquartile range. The statistical significance was set at $\mathrm{p}<0.05$. All analyses were carried out in $\mathrm{R}$ for Windows (version 3.1.2).

\section{Results}

Studies on the mechanism associated with the dysfunction of hemostatic process during the formation of AAA are the subject of great interest and intense research. The role of TF as a prognostic factor in AAA rupture was previously studied by Skóra and co-workers revealing higher levels of TF and TFPI in AAA patients [6]. We have also found elevated levels of TF and TFPI in AAA patients compared to the reference group (Table 2 ), indicating activation of coagulation process in the aorta wall. To determine whether levels of TF and TFPI can be prognostic during perioperative period we have taken into consideration a number clinical factors, such as accompanying diseases and risk factors that could influence TF and TFPI concentration changes during operative treatment. We have found that most of risk factors and associated illnesses in AAA patients did not affect TF levels, except for lower limb ischaemia. The TFPI levels are significantly altered in patients with BMI $>25$, thick thrombus, lower limb ischaemia and ischemic heart disease (Table 1). Also, we investigated whether the type of surgery selected (OAR versus EVAR) or stent graft implanted (Cook Zenith versus Gore Excluder) is related to changes in TF and TFPI.

Higher levels of TF were found in patients qualified for open surgery compared with those enrolled for EVAR (Figure 1 and Table 3) while TFPI levels were unaffected (Table 3). We found the type of EVAR implanted stent grafts had no effect of TF and TFPI level (Table 3). We have used two types of stent graftsExcluder Gore and Zenith Cook, both are aortobiiliac systems and their choice was completely random. It is worth noting that TF and TFPI levels were not analyzed before and after AAA repair, hence these data are published for the first time.

After surgery, 13 cases of early complications were reported. There were 9 cases of thromboembolic complications, 1 hemorrhagic complication and one case of respiratory tract infection. Patients undergoing open surgery had higher rates of thromboembolic

Table 2: The comparison of TF and TFPI levels patients with AAA (test group) and reference group.

\begin{tabular}{|lcccc|} 
& \multicolumn{3}{c}{ TF } & \multicolumn{2}{c|}{ TFPI } \\
\cline { 2 - 5 } & AAA patients & Reference group & AAA patients & Reference group \\
\hline Number-n & 62 & 53 & 62 & 53 \\
\hline Average-M & 219,24 & 149,17 & 77,03 & 83,87 \\
\hline SD & 120,71 & 76,42 & 27,58 & 20,72 \\
\hline Median-Me & 204,50 & 137,00 & 72,00 & 84,00 \\
\hline Quartile 25-75 p & $139,00-264,00$ & $96,50-197,75$ & $51,00-103,00$ & $69,75-97,00$ \\
\hline Min-Max & - & $18-290$ & - & $39-121$ \\
\hline
\end{tabular}


complications, particularly acute myocardial infarction and acute ischemia. The TF was significantly higher in this group, while the concentration TFPI was not affected (Figure 2 and Table 4). We have also found a strong correlation between the thickness of the anterior thrombosis in the aneurysm and the increase in plasma concentration of TFPI (Table 5).

\section{Discussion}

The hemorrhagic lesions occur after arterial endothelium damage results in the release of TF, which is the main initiator of the coagulation process, into

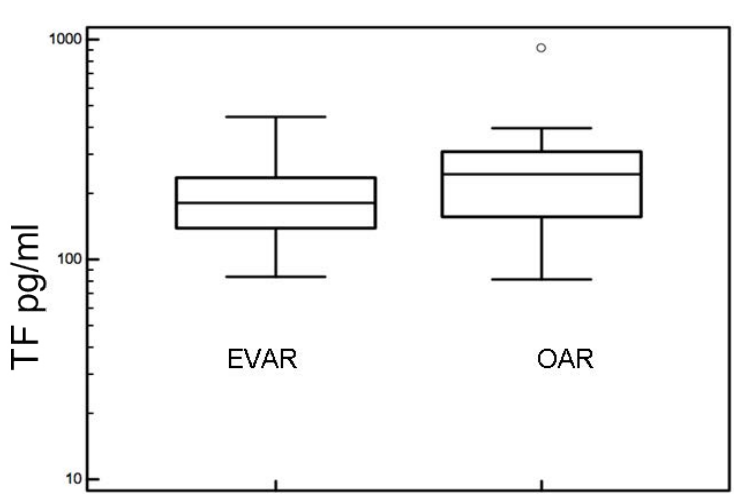

Figure 1: The concentration of TF in AAA patients before EVAR/ OAR type of surgical intervention. Statistical analysis calculated using Friedman multiple-repetition test using the Conover method. the bloodstream [7-9] and elevated TF in plasma is the evidence of the state of hyper coagulation. Previously Hobbs and co-workers showed the elevated level of TF in patients with ruptured AAA when compared to asymptomatic patients without concurrent differences in TFPI levels [10]. However, the analysis of TF and TFPI levels in context of postoperative complications has not been presented before. The elevated levels of TF, but not TFPI, were consistently observed in AAA patients suffering from acute arteriosclerosis and ischemia. Due to technical difficulties in implanting the stent graft system in these patients they were

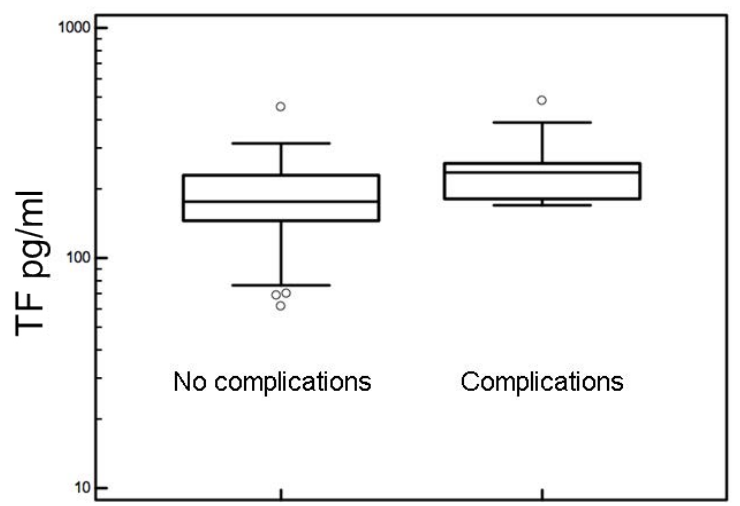

Figure 2: The differences in concentration of TF 24 hours after surgery intervention in patients with and without post-surgical complications. Statistical analysis carried out using Friedman multiple-repetition test using the Conover method.

\begin{tabular}{|c|c|c|c|c|}
\hline & \multicolumn{2}{|c|}{ TF } & \multicolumn{2}{|c|}{ TFPI } \\
\hline & $\begin{array}{l}\text { Excluder-Gore/Zenith- } \\
\text { Cook }\end{array}$ & EVAR/OAR & $\begin{array}{l}\text { Excluder-Gore/Zenith- } \\
\text { Cook }\end{array}$ & EVAR/OAR \\
\hline before & $p=0,094$ & $\mathrm{p}=0,042^{*}$ & $p=0,46$ & $p=0,94$ \\
\hline after & $p=0,42$ & $p=0,73$ & $p=0,66$ & $p=0,97$ \\
\hline $24 \mathrm{~h}$ after & $p=0,91$ & $p=0,95$ & $p=0,87$ & $p=0,37$ \\
\hline
\end{tabular}

Table 4: The concentration of TF and TFPI 24 hours after surgery in patients with post-surgical complications.

\begin{tabular}{|lccc|}
\hline \multicolumn{4}{c|}{ Surgery complications $24 \mathrm{~h}$ after } \\
\hline Measured parameter & $\begin{array}{c}\text { no complications } \\
\mathrm{n}=49\end{array}$ & $\begin{array}{c}\text { complications } \\
\mathrm{n}=13\end{array}$ & \\
\hline TF pg/ml & $183,109 \pm 73,955$ & $252,000 \pm 98,682$ & $\mathrm{p}<0,013^{*}$ \\
\hline TFPl ng/ml & $90,269 \pm 55,747$ & $97,100 \pm 39,731$ & $\mathrm{p}=0,39$ \\
\hline
\end{tabular}

Table 5: The significant correlation between the size of the aneurysm and the thickness of the thrombus and the concentration of tissue factor and tissue factor pathway inhibitor (TFPI). Statistical analysis calculated using Spearman correlation.

\begin{tabular}{|l|c|c|c|}
\hline AAA & Thrombus & TF & TFPI \\
& $3,36 \pm 1,64$ & $213 \pm 135 \mathrm{pg} / \mathrm{ml}$ & $82,1 \pm 54,5$ \\
\hline diameter $5,87 \pm 1,39 \mathrm{~cm}$ & $\mathrm{rs}=0,706^{*}$ & $\mathrm{rs}=0,06$ & $\mathrm{rs}=0,426^{*}$ \\
& $\mathrm{p}<0,0001$ & $\mathrm{p}=0,680$ & $\mathrm{p}<0,0004$ \\
\hline
\end{tabular}


mostly qualified for open. Patients undergoing open surgery also displayed higher incidence of post-surgical complications.

Higher levels of TF observed in patients undergoing open surgery may be explained by thick-er thrombus also associated with advanced atherosclerosis and ischemia. As the diameter of the aneurysm increases so does the thickness of the thrombus. The results reported by Parra and co-workers found that the thickness of the thrombosis affects the rate of enlargement of the aortic aneurysm [11]. Previous studies have confirmed a relationship between the diameter of the aneurysm and the thickness of the thrombus [12,13]. During open surgery, thrombus that shows the highest TF activity is removed from the aneurysmal lumen and its wall reconstructed using a vascular prosthesis. This may explain the reduction of post-surgical levels of TF in OAR. Also, we are first to demonstrate the association between an elevated level of TFPI and the thickness of the thrombus. No correlation was found between TF and TFPI levels and the type of stent grafts (Zenith and Excluder) implanted.

\section{Conclusion}

We have demonstrated that the concentration of TF is higher in patients undergoing open surgery and displaying post-surgical complications. Concentrations of the TFPI are higher in patients with larger aneurysms and thicker thrombosis. In summary, the monitoring of TF and TFPI levels is relevant in patients with ischemic diseases qualified for open type surgery.

\section{Acknowledgement}

This publication is part of the project WrovascIntegrated Cardiovascular Centre within Innovative Economy Operational Program, 2007-2013. POIG.01.01.02-02-001/08. The publication was supported by Wroclaw Center of Biotechnology, the Leading National Research Centre (KNOW) program for years 2014-2018.

\section{Executive summary}

Objectives: We examined plasma concentrations of essential regulators of coagulation cascade, tissue factor (TF) and tissue factor inhibitor pathway (TFPI) in patients with abdominal aortic aneurysm (AAA) during two types of surgical repair , open (OAR) and endovascular repair (EVAR).

Background: The interplay between TF and TFPI is important for the proper functioning of coagulation cascade. Although abnormal coagulation is known to contribute to the formation of AAA, little is known about the prognostic value of TF and TFPI levels in the perioperative stage of AAA repair.

Methods: We have selected the reference group of 53 healthy, first-time blood donors and 66 patients scheduled for AAA repair using EVAR or OAR and measured TF and TFPI in their blood plasma using ELISA (enzyme-linked immunosorbent assay) before surgery, immediately after and 24 hours after surgery.

Results: Patients with AAA had elevated levels of TF $(219.242 \mathrm{pg} / \mathrm{ml})$ compared to the reference group (149.170 pg/ml). TF levels were higher in AAA patients with limb ischemia $(p=0.0026)$ and mural thrombi $>30 \mathrm{~mm}(p=0.0015)$ but decreased in patient with $B M I>25(p=0.027)$. No differences were observed in TF and TFPI levels during EVAR and OAR or de-pending on the stent graft type. However, there was a correlation between the TF level and occurrence of post-surgical complications in OAR type of surgery

Conclusions: Our results suggest that monitoring levels of TF and TFPI in perioperative peri-od would be useful in risk assessment of surgery complications, especially in AAA patients with ischemic disorders undergoing OAR surgery.

\section{References}

1. Thompson RW, Geraghty PJ, Lee JK. Abdominal aortic aneurysms: basic mechanisms and clinical implications. Curr. Probl. Surg. 39: 110-230 (2002).

2. Michel JB, Martin-Ventura JL, Egido J, et al. Novel aspects of the pathogenesis of aneurysms of the abdominal aorta in humans. Cardiovasc. Res. 90: 18-27 (2011).

3. Piechota-Polanczyk A, Jozkowicz A, Nowak W, et al. The Abdominal Aortic Aneurysm and Intraluminal Thrombus: Current Concepts of Development and Treatment. Front. Cardiovasc. Med. 2: 19 (2015).

4. Parry DJ, Al-Barjas HS, Chappell L, Rashid T, Ariëns RA, Scott DJ. Hemostatic and fibrinolytic factors in men with a small abdominal aortic aneurysm. Br. J. Surg. 96: 870-877 (2009).

5. Strukova S. Coagulation-dependent inflammation and inflammation-dependent thrombosis. Front. Biosci. 11: 59-80 (2006).

6. Skóra J, Dawiskiba T, Zaleska P, et al. Prognostic value of tissue factor in patients with abdominal aortic and iliac arterial aneurysms - preliminary study. Arch. Med. Sci. 9: 1071-1077 (2013).

7. Sidloff DA, Stather PW, Choke E, Bown MJ, Sayers RD. A systematic review and meta-analysis of the association between markers of hemostasis and abdominal aortic aneurysm presence and size. J. Vasc. Surg. 59: 528-535 (2014).

8. Abdelhamid MF, Davies R, Adam D, Vohra R, Bradbury 
A. Changes in thrombin generation, fibrinolysis, platelet and endothelial cell activity, and inflammation following endovascular abdominal aortic aneurysm repair. J. Vasc. Surg. 55: 41-46 (2012).

9. Witkiewicz W, Grendziak R, Dubis J, et al. Selected clotting factors in blood of patients with abdominal aortic aneurysms. Kardiol. Pol. 70: 574-579 (2012).

10. Hobbs SD, Haggart P, Fegan C, Bradbury AW, Adam DJ. The role of tissue factor in patients undergoing open repair of ruptured and nonruptured abdominal aortic aneu-rysms. J. Vasc. Surg. 46: 682-686 (2007).
11. Parr A, McCann M, Bradshaw B, et al. Thrombus volume is associated with cardiovascular events and aneurysm growth in patients who have abdominal aortic aneurysms. J. Vasc. Surg. 53: 28-35 (2011).

12. Kotschy M, Kotschy D, Witkiewicz W. Rola czynnika tkankowego i jego inhibitora w procesie krzepnięcia krwi oraz powikłań zakrzepowych. Kardiol. Pol. 10: 1159-1162 (2010).

13. Siennicka A, Drozdzynska M, Chelstowski K, Cnotliwy M, Jastrzebska M. Haemo-static factors and intraluminal thrombus thickness in abdominal aortic aneurysm. Is sec-ondary fibrinolysis relevant? J. Physiol. Pharmacol. 64: 321-330 (2013). 